\title{
Comparative clinical evaluation of atlas and deep-learning-based auto-segmentation of organ structures in liver cancer
}

Sang Hee Ahn' ${ }^{1}$ Adam Unjin Yeo ${ }^{2}$, Kwang Hyeon Kim¹, Chankyu Kim', Youngmoon Goh³, Shinhaeng Cho ${ }^{4}$, Se Byeong Lee ${ }^{1}$, Young Kyung Lim¹', Haksoo Kim¹, Dongho Shin ${ }^{1}$, Taeyoon Kim¹, Tae Hyun Kim¹, Sang Hee Youn ${ }^{1}$, Eun Sang $\mathrm{Oh}^{1}$ and Jong Hwi Jeong ${ }^{1 *}$

\begin{abstract}
Background: Accurate and standardized descriptions of organs at risk (OARs) are essential in radiation therapy for treatment planning and evaluation. Traditionally, physicians have contoured patient images manually, which, is time-consuming and subject to inter-observer variability.

This study aims to a) investigate whether customized, deep-learning-based auto-segmentation could overcome the limitations of manual contouring and b) compare its performance against a typical, atlas-based auto-segmentation method organ structures in liver cancer.
\end{abstract}

Methods: On-contrast computer tomography image sets of 70 liver cancer patients were used, and four OARs (heart, liver, kidney, and stomach) were manually delineated by three experienced physicians as reference structures. Atlas and deep learning auto-segmentations were respectively performed with MIM Maestro 6.5 (MIM Software Inc., Cleveland, $\mathrm{OH}$ ) and, with a deep convolution neural network (DCNN). The Hausdorff distance (HD) and, dice similarity coefficient (DSC), volume overlap error (VOE), and relative volume difference (RVD) were used

to quantitatively evaluate the four different methods in the case of the reference set of the four OAR structures.

Results: The atlas-based method yielded the following average DSC and standard deviation values (SD) for the heart, liver, right kidney, left kidney, and stomach: $0.92 \pm 0.04$ (DSC \pm SD), $0.93 \pm 0.02,0.86 \pm 0.07,0.85 \pm 0.11$, and $0.60 \pm 0.13$ respectively. The deep-learning-based method yielded corresponding values for the OARs of $0.94 \pm$ $0.01,0.93 \pm 0.01,0.88 \pm 0.03,0.86 \pm 0.03$, and $0.73 \pm 0.09$. The segmentation results show that the deep learning framework is superior to the atlas-based framwork except in the case of the liver. Specifically, in the case of the stomach, the DSC, VOE, and RVD showed a maximum difference of 21.67, 25.11, 28.80\% respectively.

Conclusions: In this study, we demonstrated that a deep learning framework could be used more effectively and efficiently compared to atlas-based auto-segmentation for most OARs in human liver cancer. Extended use of the deep-learning-based framework is anticipated for auto-segmentations of other body sites.

Keywords: Contouring, Atlas-based auto-segmentation, Deep-learning-based auto-segmentation, Deep convolution neural network (DCNN)

\footnotetext{
* Correspondence: jonghwi@ncc.re.kr

'Department of Radiation Oncology, Proton Therapy Center, National Cancer

Center, 323, Ilsan-ro, Ilsandong-gu, Goyang-si, Gyeonggi-do 10408, South

Korea

Full list of author information is available at the end of the article
}

(c) The Author(s). 2019 Open Access This article is distributed under the terms of the Creative Commons Attribution 4.0 International License (http://creativecommons.org/licenses/by/4.0/), which permits unrestricted use, distribution, and reproduction in any medium, provided you give appropriate credit to the original author(s) and the source, provide a link to the Creative Commons license, and indicate if changes were made. The Creative Commons Public Domain Dedication waiver (http://creativecommons.org/publicdomain/zero/1.0/) applies to the data made available in this article, unless otherwise stated. 


\section{Background}

Accuracy and precision of the delineated target volumes and surrounding organs at risk (OARs) is critical in radiotherapy treatment processing. However, to-this-date, these segmentation-based delineations are completed manually by physicians in the majority of clinical cases, which is a time-consuming task associated with an increased workload. Consequently, the reproducibility of this process is not always guaranteed, and ultimately depends on the physician's experience [1]. In addition, manual re-segmentation is often necessary owing to anatomical changes and/or tumor responses over the course of the radiotherapy.

As such, model-based [2,3] and atlas-based [4-7] auto-segmentation methods have been developed to maximize the efficiency gain, and concurrently minimize inter-observer variation. Various modelbased methods have been published. Specifically, Qazi et al. [3] demonstrated use of adaptive model-based auto-segmentation of the normal and target structures for the head and neck, and Chen et al. [2] showed that active shape model-based segmentation could yield accuracy improvements of the order of $10.7 \%$ over atlas-based segmentation for lymph node regions. In the last few years, machine learning technology has been actively applied to various medical fields, such as for cancer diagnosis [8-10], medical imaging [11], radiation treatment $[11,12]$, and pharmacokinetics [13]. The application of one of the deep learning models [14], the convolutional neural network (CNN) [15], has recently yielded remarkable results in medical image segmentation [16-20].

The main advantage of deep learning methods is that they automatically generate the most suitable model from given training datasets. Therefore, a comparative study of the accuracy of each model is required to use auto-segmentation in clinical practice. Recently, Lustberg et al. [21] compared the auto contouring results in five organ structures with the use of the prototype of a commercial deep-learning contouring program (Mirada DLC Expert, Mirada Medical Ltd., Oxford, United Kingdom) with those obtained from an atlasbased contouring program (Mirada Medical Ltd., Oxford, United Kingdom).

In this study, we used the open source deep learning library, Keras (where the model can be loaded into the
Tensorflow backend) instead of the commercial program. In addition, our neural network is based on $\mathrm{Fu}$ sion net, an extension of the U-net suitable for medical image segmentation. This study aims to evaluate the clinical feasibility of an open source deep learning framework, using 70 liver cancer patients by comparing its performance against a commercially available atlas-based auto-segmentation framework.

\section{Methods}

\section{Clinical datasets}

Seventy patients with liver cancer diagnosed at the $\mathrm{Na}$ tional Cancer Center in South Korea between the year of 2016-2017 were included in this study. All patients were treated with proton therapy, using 10 fractions of 660 or $700 \mathrm{cGy}$, with respective total doses of $6600 \mathrm{cGy}$ and $7000 \mathrm{cGy}$. The characteristics of the patients are listed in Table 1. All computer tomography (CT) images were acquired using a General Electric (GE) Light speed radiotherapy (RT) system (GE Medical Systems, Milwaukee, WI). We used abdominal CT images with, the following dimensions for each axial slice: image matrix $=512 \times 512$, slice numbers $=80-128$, pixel spacing $=1.00-1.04 \mathrm{~mm}$, and slice thickness $=2.50 \mathrm{~mm}$. Manually segmented contours for each organ were delineated by three senior expert physicians, and included segmentations of the heart, liver, kidney (left, right), and stomach. Manually segmented contours included the organ contours of the heart, liver, kidney (left, right), and stomach, which were mutually accepted by the three senior physicians following a joint discussion.

The study protocol conformed to the ethical guidelines of the Declaration of Helsinki as revised in 1983, and was approved by institutional review board (IRB) of National Cancer Center without IRB number. All patient data has been fully anonymized, and all methods were performed in accordance with the relevant guidelines and regulations outlined by our institution.

\section{Deep convolutional neural network}

The network used was based on the open-source library Keras (version 2.2.4) [22] and the reference implementation of Fusion Net [23]. This network is a deep neural network which was developed based on

Table 1 Patient characteristics in this study

\begin{tabular}{|c|c|c|c|c|c|c|c|c|c|c|c|c|}
\hline \multirow[t]{2}{*}{ Patients } & \multirow[t]{2}{*}{ Male } & \multirow[t]{2}{*}{ Female } & \multirow{2}{*}{$\begin{array}{l}\text { Average } \\
\text { age }\end{array}$} & \multicolumn{9}{|c|}{ Location of liver cancer lesion } \\
\hline & & & & S1 & $\mathrm{S} 2$ & S3 & S4 & S5 & S6 & S7 & S8 & M \\
\hline Training set & 52 & 8 & 67 & 2 & 1 & 3 & 6 & 6 & 2 & 5 & 24 & 11 \\
\hline Testing set & 8 & 2 & 69 & - & - & 1 & 1 & 1 & 1 & - & 3 & 3 \\
\hline
\end{tabular}


the application of a residual $\mathrm{CNN}$ as an extension of U-net [24] to enable more accurate end-to-end image segmentation. It consists of a down-sampling (encoding) path and an up-sampling (decoding) path, as shown in Fig. 1. On the encoding path, we used a residual block layer (three convolution layer and one skip connection) between the two $3 \times 3$ convolution layers. Each of these layers was followed by a rectified linear unit (ReLu) [25], and one maximum pooling. On the decoding path, we used a $2 \times 2$ transposed convolution and a residual block layer between the two $3 \times 3$ convolution layers followed by a ReLu activation function. To avoid overfitting during the training stage, batch normalization [26] and dropout [27] were added to the layers. In the final layer, we used a $1 \times 1$ convolution network with a sigmoid activation function and a dice similarity coefficient loss function [28]. We used Adam [29] as an optimizer with the following training parameters: a learning rate of $1.0 \mathrm{E}-05$, mini-batch size of twelves images, and a weight decay. A more detailed specification of our deep neural network, such as the number of feature maps, their sizes and ingredients, are listed Table 2. The experiments were conducted on a computer workstation with an Intel i7 central processing unit (CPU) with a $24 \mathrm{~GB}$ main memory, and a computer unified device architecture (CUDA) library on the graphics processing unit (GPU) (NVIDIA GeForce TITAN-Xp with $12 \mathrm{~GB}$ of memory). Network training of the deep convolutional neural network (DCNN) took approximately 48 h to run 2000 epochs on the training and validation datasets.

\section{Segmentation image preprocessing}

CT planning images from patients and the required contouring information used for training of the DCNN were obtained using the Eclipse planning software (version 13.6, Varian Oncology Systems, Palo Alto, CA, USA). All $\mathrm{CT}$ images were converted to grayscale images, and the contouring points were converted to segmented label images in a binary format, as shown in Fig. 2. Hounsfield unit (HU) values were windowed in the range of - 100600 to exclude irrelevant organs All images were downsampled from the conventional size of $512 \times 512$ pixels to the size of $256 \times 256$ pixels owing to graph card memory resource limitations and reduced DCNN training time constraints.

\section{Deep-learning-based segmentation}

The deep-learning-based segmentation process consisted of three steps. The first was the random separation into training and validation sets consisting of 45 and $15 \mathrm{pa}-$ tient datasets, respectively, and the preprocessing and

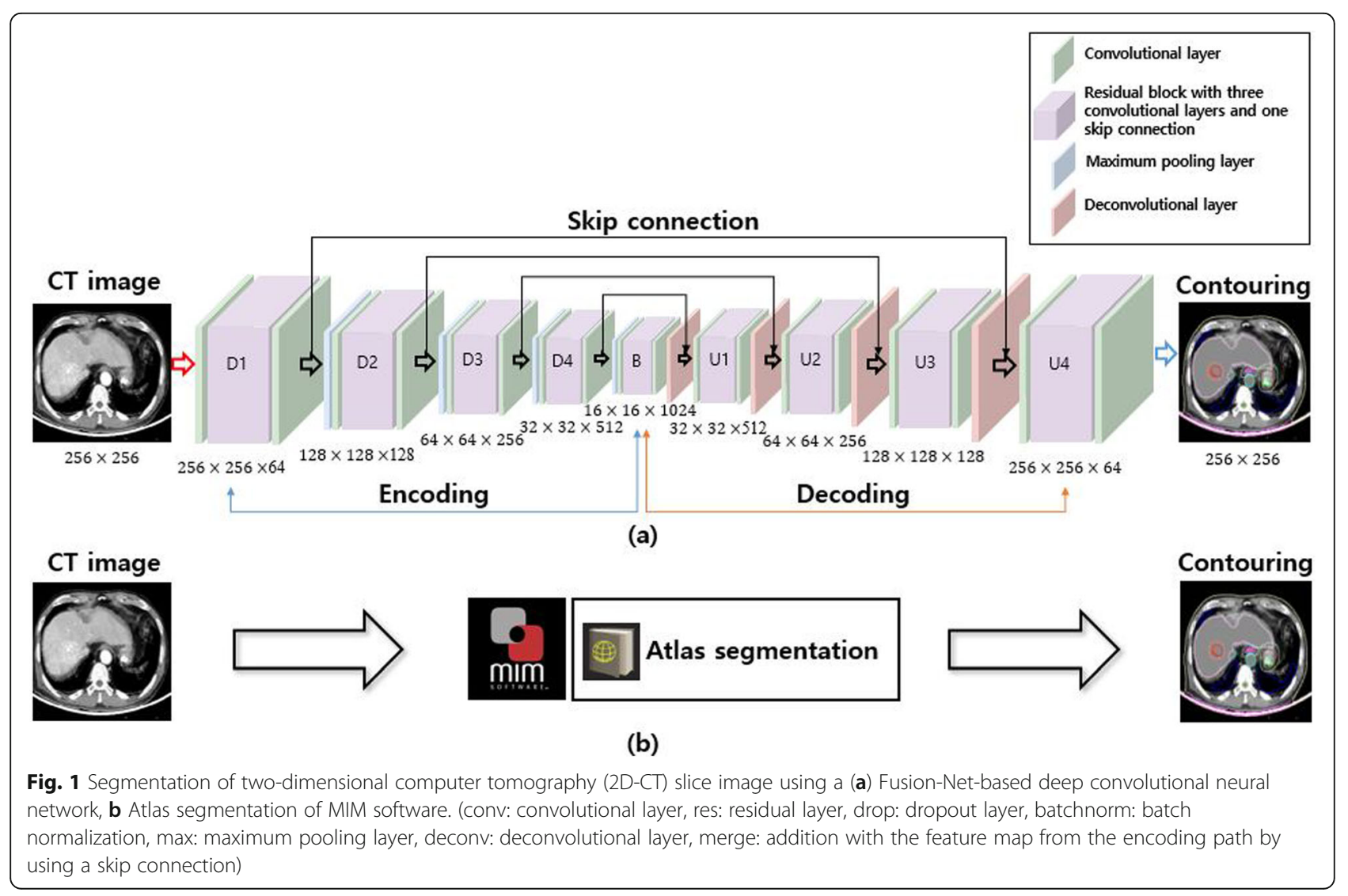


Table 2 Architecture of the proposed convolutional neural network

\begin{tabular}{lll}
\hline Block type & Ingredients & Size of feature maps \\
\hline Input & - & $256 \times 256 \times 1$ \\
Down layer (D1) & conv+res + drop+conv+batchnorm+max & $128 \times 128 \times 64$ \\
Down layer (D2) & conv+res + drop+conv+batchnorm+max & $64 \times 64 \times 128$ \\
Down layer (D3) & conv+res + drop+conv+batchnorm+max & $32 \times 32 \times 256$ \\
Down layer (D4) & conv+res + drop+conv+batchnorm+max & $16 \times 16 \times 512$ \\
Bridge layer (B) & conv+res+ conv & $16 \times 16 \times 1024$ \\
Upscaling layer (U1) & deconv+merge+conv+res + conv & $32 \times 32 \times 512$ \\
Upscaling layer (U2) & deconv+merge+conv+res + conv & $64 \times 64 \times 256$ \\
Upscaling layer (U3) & deconv+merge+conv+res + conv & $128 \times 128 \times 128$ \\
Upscaling layer (U4) & deconv+merge+conv+res + conv & $256 \times 256 \times 64$ \\
Output & conv & $256 \times 256 \times 1$ \\
\hline
\end{tabular}

preparation of 10 independent test dataset images for the deep convolutional neural network.

In the second step, we trained the DCNN using the training datasets for each of the organs. In the final step, the test image set was segmented into a test dataset with DCNN (Fig. 3).

\section{Atlas-based-segmentation}

Atlas-based segmentation is a method used to locate the interface between the test image and the optimally matched organs from labeled, segmented reference image data [30].

The commercial atlas-based contouring software MIM Maestro 6.5 (MIM Software Inc., Cleveland, $\mathrm{OH}$, USA) was used to generate the contours of the ten patients automatically test datasets for the OARs. Segmentation processing was performed on a single organ basis instead of multiple organ segmentation, and the outcomes were compared with those of the deep-

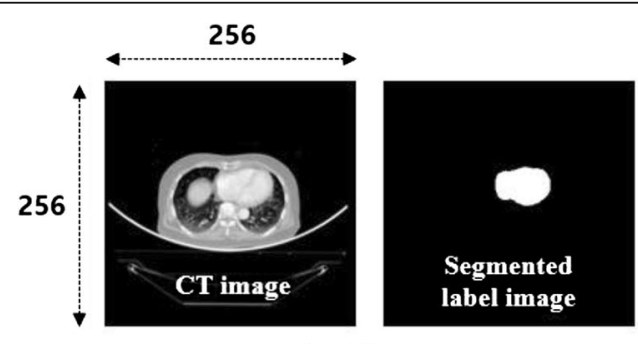

(a) Heart

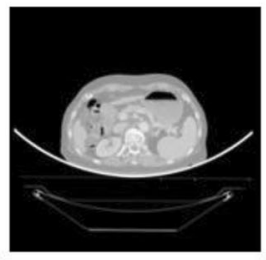

(c) Right Kidney

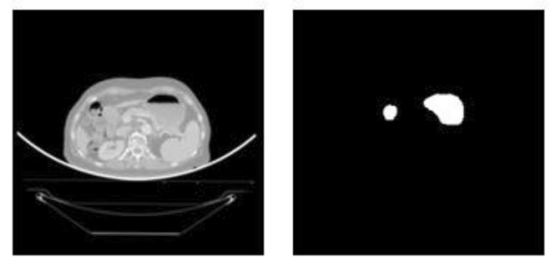

(e) Stomach

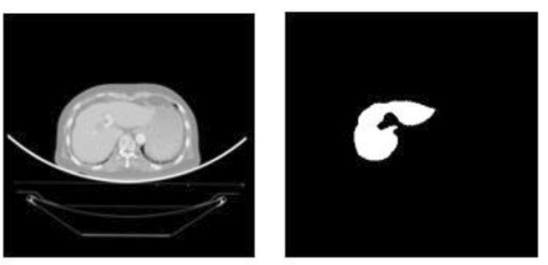

(b) Liver

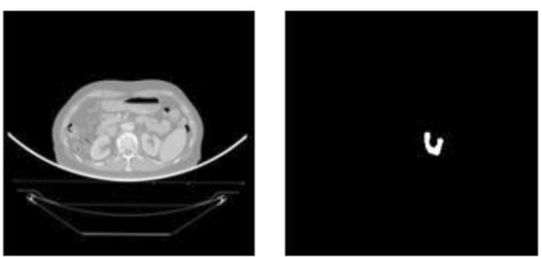

(d) Left Kidney

DCNN model learning 


\section{Step one}

DCNN training with 60 patients

(45 training, 15 validation datasets)

CT images (HU windowing)

\section{Step two}

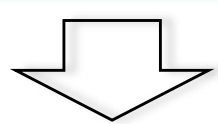

Test data10 patients CT images (HU windowing)

of heart, liver, kidney and stomach segmentation

\section{Step three}

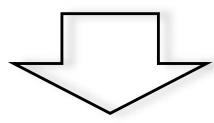

\section{Evaluation of auto segmentation results}

Fig. 3 Work flowchart for deep convolution neural network (DCNN) training and testing

learning-based segmentation conducted using the same conditions. We used MIM supported label fusion algorithms based on the majority vote (MV) algorithm.

For segmentation, a training set with data from 60 patients was registered to the MIM Maestro 6.5 atlas library with CT planning images alongside the respective manual contours of the heart, liver, kidney, and stomach. The slice thicknesses of the CT images were not changed during their registration with the atlas library.

\section{Quantitative evaluations of auto-segmentation}

To quantitatively evaluate the accuracy of deep learning and atlas-based auto-segmentations, the Dice similarity coefficient (DSC) and Hausdorff distance (HD) were used for quantitative analyses on accuracy [31]. The DSC method, calculates the overlapping results of two different volumes according to the equation,

$$
D S C(\text { dice similarity coefficient })=\frac{2|A \cap B|}{|A|+|B|},
$$

where $\mathrm{A}$ is the manual contouring volume, and $\mathrm{B}$ is the auto-segmentation volume (deep learning and atlas segmentation results). DSC takes values between zero and one. When the DSC value approaches zero, the manual and auto-segmentation outcomes differ significantly. However, as the DSC value approaches unity, the twovolumes exhibit increased similarities.

The second method is the HD method. After calculating the Euclidean distance of the surfaces of each contour point between A and B, the similarity of A and B is determined according to the distance of the nearest maximum distance. HD is thus defined as,

$$
\begin{aligned}
& \text { Hausdorff distance }(H D) \\
& \quad=\max (h(A, B), h(B, A)),
\end{aligned}
$$

where $\mathrm{h}(\mathrm{A}, \mathrm{B})$ is the directed $\mathrm{HD}$ from $\mathrm{A}$ to $\mathrm{B}$ and is given by.

$$
h=\max (\min (\|a-b\|))
$$

$$
\mathrm{a} \in \mathrm{A}, \mathrm{b} \in \mathrm{B}
$$

As the HD approaches zeros, the difference between the manual contouring and auto contouring becomes smaller. By contrast, if the coefficient is greater than zero, the similarity between the two volumes decreases. 
The third method is the volume overlap error (VOE) [32]. VOE can be calculated by subtracting the Jaccard coefficient from the value of unit by comparing dissimilarities between the two volumes.

$$
\text { VOE (volume overlap error) }=1-\frac{|A \cap B|}{|A \cup B|},
$$

The last method is the relative volume difference (RVD) [32]. RVD compares the sizes between two volumes.

$$
\operatorname{RVD}\left(\text { relative volume difference) }=\frac{|B|-|A|}{|A|},\right.
$$

Contrary to DSC, as the VOE and RVD approach zero, the manual contouring and auto contouring volumes only yield small volume differences, and values larger than zero reduce the similarity between the two volumes.

\section{Results}

For quantitative evaluations, the DSC and HD were calculated for each test dataset, and the results are shown in Tables 3 and 4. For qualitative visual assessment, Fig. 4 shows, a specific patient case where the three delineation methods are compared, i.e., the atlasbased $\left(C_{\text {atlas }}\right)$, deep-learning-based $\left(C_{\text {deep }}\right)$, and manual contouring methods $\left(C_{\text {manual }}\right)$. In all the organ cases studied herein (i.e., heart, liver, kidney, and stomach), the $C_{\text {deep }}$ results more accurate matched to the $C_{\text {manual }}$ compared to the $C_{\text {atlas }}$ results. However, both $C_{\text {atlas }}$ and $C_{\text {deep }}$ were not excluded in the hepatic artery region (Fig. 4, red arrow). For the kidney case, neither the $C_{\text {atlas }}$ nor the $C_{\text {deep }}$ outcomes differed significantly from the evoked $\mathrm{C}_{\text {manual }}$ outcomes from DSC.

The methods of auto-segmentation were quantitatively compared using DSC, HD, VOE and RVD metrics against manual contours (i.e., the reference), and are presented in Tables 3, 4, 5, and 6, respectively.

The average DSC values $\left( \pm\right.$ SD) of $C_{\text {atlas }}$ are $0.92( \pm$ $0.04), 0.93$ ( \pm 0.02$), 0.86( \pm 0.07), 0.85( \pm 0.11)$, and $0.60( \pm$ 0.13 ) for the heart, liver, right kidney, left kidney, and stomach, respectively. The respective outcomes for the same DSC analyses for $C_{\text {deep }}$ are $0.94( \pm 0.01), 0.93( \pm$ $0.01), 0.88( \pm 0.03), 0.86( \pm 0.03)$, and $0.73( \pm 0.09)$, for the heart, liver, right kidney, left kidney, and stomach, respectively.

The HD values $( \pm \mathrm{SD})$ for $\mathrm{C}_{\text {atlas }}$ are $2.16( \pm 1.52) \mathrm{mm}$, $2.23( \pm 0.81) \mathrm{mm}, 1.78( \pm 1.34) \mathrm{mm}, 1.90( \pm 1.24) \mathrm{mm}$, and $6.76( \pm 2.31) \mathrm{mm}$, for the heart, liver, right kidney, left kidney, and stomach, respectively. The respective outcomes for the HD values based on the same analysis for $C_{\text {deep }}$ are $1.61( \pm 0.28) \mathrm{mm}, 2.17( \pm 0.39) \mathrm{mm}$, $1.61( \pm 0.52) \mathrm{mm}, 1.88( \pm 0.31) \mathrm{mm}$, and $4.86( \pm 1.57)$ $\mathrm{mm}$, for the heart, liver, right kidney, left kidney, and stomach, respectively, as shown in Fig. 6. The average DSC outcomes for $C_{\text {deep }}$ are higher in all the cases except for the liver. Specifically, there was a maximum difference of $21.67 \%$ in the stomach case, as shown in Table 8. It is important to note that the standard deviations of the DSC values for $C_{\text {atlas }}$ were higher than those of $C_{\text {deep }}$ for all the studied structures, i.e., $C_{\text {atlas }}$ exhibits broader interquartile ranges than $C_{\text {deep }}$ in the boxplot, as shown in Fig. 5 .

The VOE and RVD results showed significant differences between $C_{\text {atlas }}$ and $C_{\text {deep }}$ compared to DSC,

Table 3 Comparison of dice similarity coefficients (DSC) obtained from atlas and deep-learning-based segmentations in the cases of

\begin{tabular}{|c|c|c|c|c|c|c|c|c|c|c|}
\hline \multirow{2}{*}{$\begin{array}{l}\text { Test } \\
\text { Case }\end{array}$} & \multicolumn{2}{|l|}{ Heart } & \multicolumn{2}{|l|}{ Liver } & \multicolumn{2}{|c|}{ Right kidney } & \multicolumn{2}{|c|}{ Left kidney } & \multicolumn{2}{|c|}{ Stomach } \\
\hline & $C_{\text {atlas }}$ & $\overline{C_{\text {deep }}}$ & $C_{\text {atlas }}$ & $C_{\text {deep }}$ & $\mathrm{C}_{\text {atlas }}$ & $C_{\text {deep }}$ & $C_{\text {atlas }}$ & $C_{\text {deep }}$ & $C_{\text {atlas }}$ & $C_{\text {deep }}$ \\
\hline$\# 1$ & 0.95 & 0.96 & 0.93 & 0.93 & 0.85 & 0.86 & 0.78 & 0.83 & 0.41 & 0.80 \\
\hline$\# 2$ & 0.93 & 0.93 & 0.92 & 0.93 & 0.93 & 0.92 & 0.93 & 0.88 & 0.78 & 0.71 \\
\hline \# 3 & 0.96 & 0.95 & 0.95 & 0.94 & 0.87 & 0.88 & 0.61 & 0.84 & 0.58 & 0.71 \\
\hline$\# 4$ & 0.96 & 0.96 & 0.95 & 0.94 & 0.85 & 0.86 & 0.93 & 0.89 & 0.53 & 0.57 \\
\hline \# 5 & 0.92 & 0.93 & 0.94 & 0.93 & 0.84 & 0.89 & 0.89 & 0.78 & 0.63 & 0.88 \\
\hline \# 6 & 0.85 & 0.94 & 0.89 & 0.92 & 0.89 & 0.89 & 0.91 & 0.88 & 0.61 & 0.79 \\
\hline$\# 7$ & 0.85 & 0.94 & 0.90 & 0.93 & 0.95 & 0.93 & 0.94 & 0.86 & 0.79 & 0.72 \\
\hline$\# 8$ & 0.86 & 0.93 & 0.92 & 0.93 & 0.91 & 0.84 & 0.92 & 0.85 & 0.74 & 0.83 \\
\hline$\# 9$ & 0.96 & 0.94 & 0.92 & 0.93 & 0.70 & 0.84 & 0.88 & 0.88 & 0.38 & 0.61 \\
\hline \# 10 & 0.91 & 0.93 & 0.94 & 0.94 & 0.78 & 0.84 & 0.70 & 0.88 & 0.56 & 0.64 \\
\hline \multirow[t]{2}{*}{ Avg SD } & 0.92 & 0.94 & 0.93 & 0.93 & 0.86 & 0.88 & 0.85 & 0.86 & 0.60 & 0.73 \\
\hline & 0.04 & 0.01 & 0.02 & 0.01 & 0.07 & 0.03 & 0.11 & 0.03 & 0.13 & 0.09 \\
\hline
\end{tabular}
the four tested organs (heart, liver, kidney, stomach). Averages and standard deviations are listed for all the ten tested cases 
Table 4 Comparison of Hausdorff distances (HD) for atlas against deep-learning-based segmentation for the with four organs (heart, liver, kidney, stomach). Averages and standard deviations are listed for ten tested cases

\begin{tabular}{|c|c|c|c|c|c|c|c|c|c|c|}
\hline \multirow{2}{*}{$\begin{array}{l}\text { Test } \\
\text { Case }\end{array}$} & \multicolumn{2}{|c|}{ Heart } & \multicolumn{2}{|l|}{ Liver } & \multicolumn{2}{|c|}{ Right kidney } & \multicolumn{2}{|c|}{ Left kidney } & \multicolumn{2}{|c|}{ Stomach } \\
\hline & $C_{\text {atlas }}$ & $C_{\text {deep }}$ & $C_{\text {atlas }}$ & $C_{\text {deep }}$ & $C_{\text {atlas }}$ & $C_{\text {deep }}$ & $C_{\text {atlas }}$ & $C_{\text {deep }}$ & $C_{\text {atlas }}$ & $C_{\text {deep }}$ \\
\hline$\# 1$ & 1.06 & 1.15 & 1.90 & 1.89 & 1.03 & 1.80 & 2.26 & 1.49 & 8.88 & 3.47 \\
\hline$\# 2$ & 1.36 & 1.88 & 1.79 & 1.72 & 1.14 & 1.29 & 0.95 & 1.51 & 3.09 & 3.57 \\
\hline \# 3 & 0.66 & 1.45 & 1.37 & 2.85 & 1.35 & 1.69 & 4.82 & 1.79 & 6.70 & 5.54 \\
\hline$\# 4$ & 0.65 & 1.23 & 2.05 & 2.16 & 0.55 & 0.52 & 0.85 & 1.58 & 7.69 & 5.35 \\
\hline \# 5 & 1.53 & 1.46 & 1.34 & 1.56 & 1.16 & 1.95 & 1.22 & 2.37 & 6.83 & 2.65 \\
\hline \# 6 & 4.70 & 1.80 & 4.09 & 2.27 & 1.59 & 1.95 & 1.33 & 2.04 & 8.35 & 5.99 \\
\hline$\# 7$ & 4.70 & 1.63 & 2.84 & 1.84 & 0.76 & 1.20 & 1.02 & 2.37 & 3.35 & 4.62 \\
\hline \# 8 & 3.47 & 1.59 & 2.06 & 2.31 & 1.46 & 1.32 & 1.00 & 1.77 & 4.58 & 3.38 \\
\hline$\# 9$ & 0.92 & 1.80 & 3.05 & 2.63 & 4.66 & 2.48 & 2.10 & 1.80 & 10.67 & 5.89 \\
\hline$\# 10$ & 2.51 & 2.10 & 1.83 & 2.45 & 4.11 & 1.91 & 3.45 & 2.10 & 7.42 & 8.14 \\
\hline \multirow[t]{2}{*}{ Avg SD } & 2.16 & 1.61 & 2.23 & 2.17 & 1.78 & 1.61 & 1.90 & 1.88 & 6.76 & 4.86 \\
\hline & 1.52 & 0.28 & 0.81 & 0.39 & 1.34 & 0.52 & 1.24 & 0.31 & 2.31 & 1.57 \\
\hline
\end{tabular}

Avg: Average

SD: Standard deviation

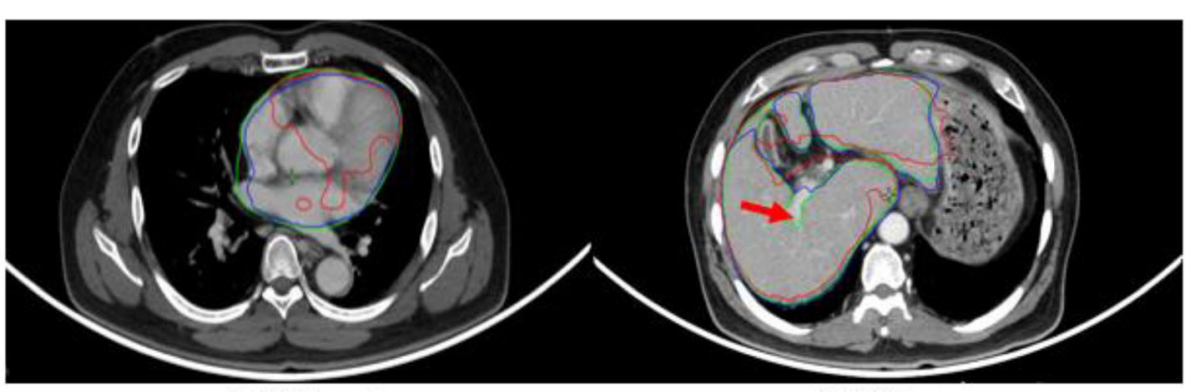

(a) Heart

(b) Liver

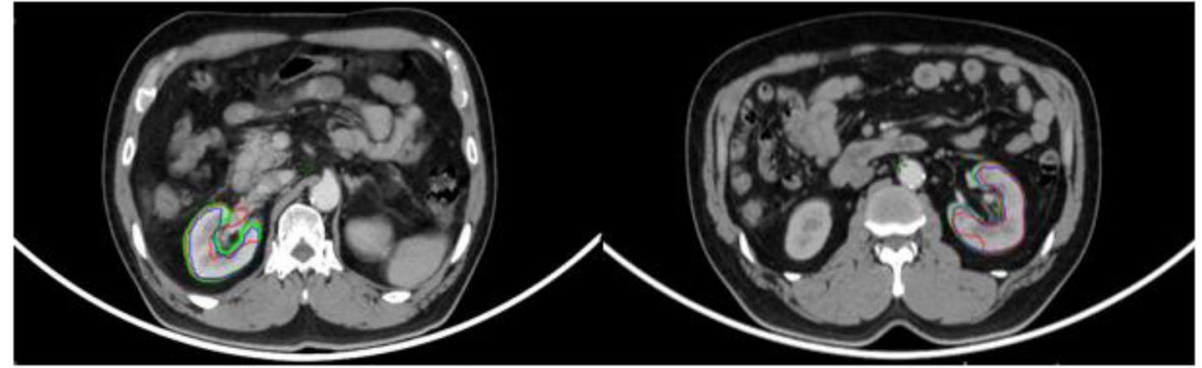

(c) Right Kidney

(d) Left Kidney

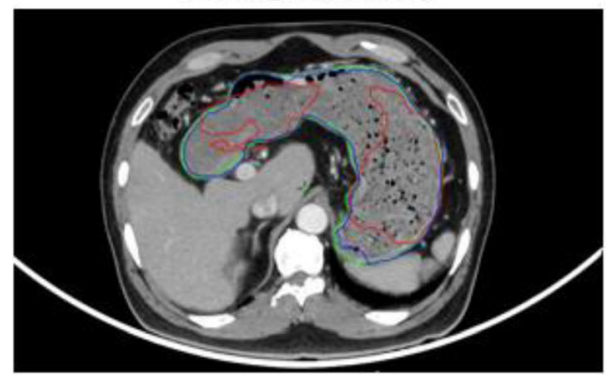

(e) Stomach

Fig. 4 Selected CT slices of one of the studied patients with a manual contour (green), atlas-based contour (red), and deep-learning-based contour (blue) for the (a) heart, (b) liver, (c) right kidney, (d) left kidney, and (e) stomach 
Table 5 Comparison of volume overlap error (VOE) for atlas-based segmentation against deep-learning-based segmentation with four organs (heart, liver, kidney, stomach). Averages and standard deviations are listed for ten test cases

\begin{tabular}{|c|c|c|c|c|c|c|c|c|c|c|}
\hline \multirow{2}{*}{$\begin{array}{l}\text { Test } \\
\text { Case }\end{array}$} & \multicolumn{2}{|l|}{ Heart } & \multicolumn{2}{|l|}{ Liver } & \multicolumn{2}{|c|}{ Right kidney } & \multicolumn{2}{|c|}{ Left kidney } & \multicolumn{2}{|c|}{ Stomach } \\
\hline & $C_{\text {atlas }}$ & $C_{\text {deep }}$ & $C_{\text {atlas }}$ & $C_{\text {deep }}$ & $C_{\text {atlas }}$ & $C_{\text {deep }}$ & $C_{\text {atlas }}$ & $C_{\text {deep }}$ & $C_{\text {atlas }}$ & $C_{\text {deep }}$ \\
\hline$\# 1$ & 8.49 & 6.83 & 10.22 & 10.58 & 12.88 & 10.31 & 30.15 & 18.37 & 84.83 & 31.01 \\
\hline$\# 2$ & 11.68 & 13.24 & 9.95 & 7.81 & 17.62 & 18.38 & 14.39 & 19.62 & 45.96 & 39.11 \\
\hline$\# 3$ & 7.34 & 11.17 & 8.39 & 13.44 & 12.42 & 11.07 & 63.90 & 12.42 & 65.73 & 34.86 \\
\hline$\# 4$ & 11.17 & 12.73 & 6.73 & 7.34 & 8.86 & 8.54 & 8.34 & 10.31 & 81.17 & 54.12 \\
\hline \# 5 & 13.87 & 11.89 & 10.29 & 11.64 & 9.39 & 7.59 & 20.76 & 35.07 & 72.42 & 31.16 \\
\hline \# 6 & 26.60 & 11.95 & 34.32 & 15.32 & 15.17 & 15.66 & 14.32 & 17.84 & 55.58 & 35.68 \\
\hline$\# 7$ & 24.39 & 6.66 & 20.41 & 11.02 & 8.10 & 9.03 & 9.99 & 12.00 & 32.21 & 41.44 \\
\hline$\# 8$ & 19.34 & 12.12 & 13.29 & 10.30 & 15.27 & 15.41 & 16.58 & 12.53 & 39.02 & 23.24 \\
\hline$\# 9$ & 7.74 & 10.34 & 13.14 & 12.07 & 40.66 & 13.06 & 20.15 & 14.59 & 85.51 & 42.75 \\
\hline \# 10 & 21.04 & 11.48 & 8.37 & 8.68 & 34.68 & 12.87 & 57.73 & 10.39 & 63.92 & 41.97 \\
\hline \multirow[t]{2}{*}{ Avg SD } & 15.17 & 10.84 & 13.51 & 10.82 & 17.51 & 12.19 & 25.63 & 16.31 & 62.64 & 37.53 \\
\hline & 6.77 & 2.18 & 7.83 & 2.36 & 10.57 & 3.33 & 18.57 & 7.01 & 18.10 & 7.99 \\
\hline
\end{tabular}

Avg: Average

SD: Standard deviation

as shown in Figs. 7 and 8. In Table 8, average of DSC results in the liver case were not different, but the VOE and RVD showed a more accurate difference of $\sim 3 \%$, and the heart, kidney (left, right) and stomach also showed significantly differences than DSC, as shown in Tables 9 and 10. In addition, Christ et al. [32] have also published a liver case auto segmentation study, whereby VOE and RVD yielded more sensitive differences compared to the DSC results.

\section{Discussion}

In this study, $70 \mathrm{CT}$ patient datasets (45 for training, 15 for validation, and 10 for testing) were used to compare the performances of the atlas-and deep-learning-based auto-segmentation frameworks. In the study of La Macchia et al. [33], the DSC results obtained from the autosegmentation analyses for the heart, liver, left kidney and right kidney, with the use of the three commercially available systems (ABAS 2.0, MIM 5.1.1, and Velocity AI 2.6.2) were in the ranges of $0.87-0.88,0.90-0.93,0.81-$

Table 6 Comparison of relative volume difference (RVD) for atlas-based segmentation against deep-learning-based segmentation with four organs (heart, liver, kidney, stomach). Averages and standard deviations are listed for ten test cases

\begin{tabular}{|c|c|c|c|c|c|c|c|c|c|c|}
\hline \multirow{2}{*}{$\begin{array}{l}\text { Test } \\
\text { Case }\end{array}$} & \multicolumn{2}{|l|}{ Heart } & \multicolumn{2}{|l|}{ Liver } & \multicolumn{2}{|c|}{ Right kidney } & \multicolumn{2}{|c|}{ Left kidney } & \multicolumn{2}{|c|}{ Stomach } \\
\hline & $C_{\text {atlas }}$ & $C_{\text {deep }}$ & $C_{\text {atlas }}$ & $C_{\text {deep }}$ & $\mathrm{C}_{\text {atlas }}$ & $C_{\text {deep }}$ & $C_{\text {atlas }}$ & $C_{\text {deep }}$ & $C_{\text {atlas }}$ & $C_{\text {deep }}$ \\
\hline \# 1 & 0.78 & 1.10 & 3.22 & 2.44 & 4.63 & 0.36 & 14.9 & 2.67 & 84.38 & 14.56 \\
\hline$\# 2$ & 7.65 & 9.33 & 12.10 & 0.89 & 3.57 & 1.77 & 6.27 & 0.12 & 21.33 & 18.52 \\
\hline \# 3 & 3.08 & 8.51 & 0.70 & 3.81 & 9.17 & 3.39 & 20.25 & 1.44 & 48.75 & 25.55 \\
\hline$\# 4$ & 7.40 & 5.13 & 3.30 & 0.89 & 1.62 & 0.51 & 0.60 & 1.26 & 59.02 & 19.12 \\
\hline \# 5 & 15.48 & 0.62 & 2.89 & 1.16 & 1.18 & 1.58 & 1.56 & 7.57 & 63.49 & 10.94 \\
\hline \# 6 & 24.82 & 8.20 & 12.20 & 0.35 & 10.58 & 1.77 & 6.16 & 1.55 & 70.37 & 43.71 \\
\hline$\# 7$ & 23.57 & 1.77 & 13.21 & 0.81 & 2.11 & 2.35 & 3.94 & 2.69 & 25.68 & 30.79 \\
\hline$\# 8$ & 11.80 & 7.51 & 0.11 & 0.83 & 4.52 & 9.72 & 12.15 & 3.51 & 21.67 & 14.32 \\
\hline$\# 9$ & 8.31 & 2.74 & 4.66 & 3.64 & 42.26 & 12.9 & 12.29 & 2.49 & 89.19 & 20.80 \\
\hline \# 10 & 26.12 & 6.75 & 3.18 & 3.81 & 17.87 & 10.93 & 24.21 & 1.21 & 16.73 & 14.30 \\
\hline \multirow[t]{2}{*}{ Avg SD } & 12.90 & 5.17 & 5.56 & 1.86 & 9.75 & 4.53 & 10.23 & 2.45 & 50.06 & 21.26 \\
\hline & 8.72 & 3.17 & 4.72 & 1.34 & 11.89 & 4.49 & 7.52 & 1.94 & 25.92 & 9.35 \\
\hline
\end{tabular}




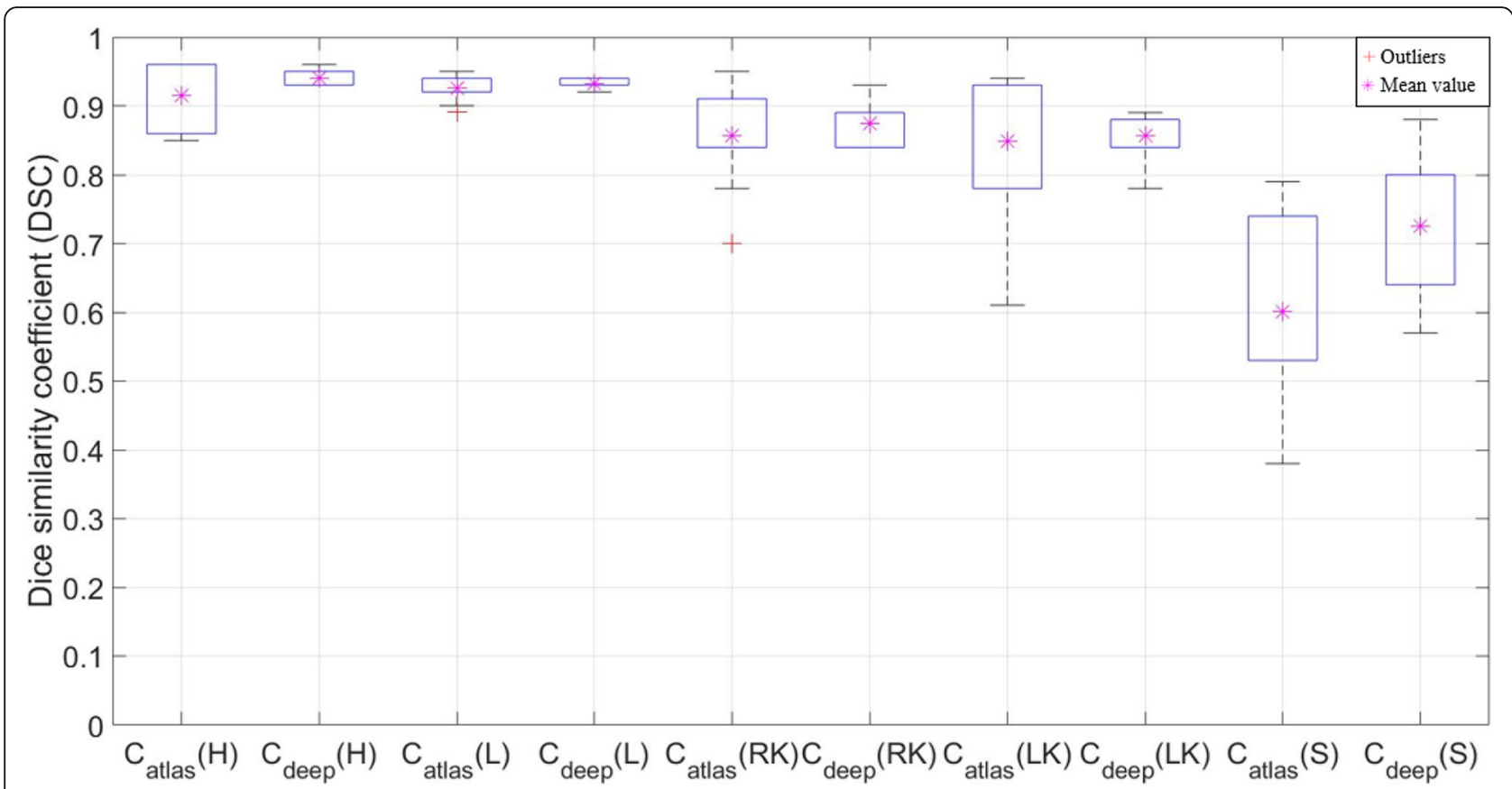

Fig. 5 Comparison of Hausdorff distances $(H D)$ for deep learning contour $\left(\mathrm{C}_{\text {deep }}\right)$ and atlas-based contour $\left(\mathrm{C}_{\text {atlas }}\right)$ segmentations for the heart $(\mathrm{H})$, liver (L), right kidney (RK), left kidney (LK), and stomach (S)

0.89 , and $0.83-0.89$, respectively. The heart yielded lower DSC scores than our reported results, whereas the other organ cases were similar to our segmented results.

However, poorer performance outcomes were evoked in the case of the stomach compared to the other organs in terms of DSC owing to the fact that the performance of our method depended on the presence of gas bubbles and on the variation of the stomach shapes among the studied patient cases (Table 8). Nevertheless, as shown in Tables 7 and 8, it is important to note that the deep learning method yielded more accurate results both in terms of the DSC (by 21.67\%) and HD (-1.90 mm) compared to the atlas-based method.

The time-efficiency was based on the average times required by the atlas and deep-learning-based segmentation methods for the four organs, which were $75 \mathrm{~s}$ and $76 \mathrm{~s}$, respectively (i.e., there was no statistically significant difference because $p$-values were larger than 0.05 when a ranked Wilcoxon test was performed).

Table 7 Differences between HD mean values associated with the deep learning and atlas-based contouring methods

\begin{tabular}{lllllll}
\hline \multicolumn{2}{l}{ Subject organs } & Heart & Liver & Right Kidney & Left Kidney & Stomach \\
\hline $\mathrm{HD}(\mathrm{mm})$ & $\mathrm{C}_{\text {deep }}$ & 1.61 & 2.17 & 1.61 & 1.88 & 4.86 \\
& $\mathrm{C}_{\text {atlas }}$ & 2.16 & 2.23 & 1.78 & 1.90 & 6.76 \\
$\mathrm{C}_{\text {deep }}-\mathrm{C}_{\text {atlas }}(\mathrm{mm})$ & -0.55 & -0.06 & -0.17 & -0.02 & -1.90 \\
\hline
\end{tabular}

However, in the case of the atlas-based segmentation, the time required for multi-organ segmentation can be reduced. A recent study by Gibson et al. [34] demonstrated a multi-organ segmentation approach using the deep learning framework. Our future studies will be undertaken based on the implementation of multiorgan segmentation using DCNN to investigate the impact of discrepancies among different segmentation methods in radiation treatment planning.

It is also important to note that this study is associated with some limitations. First, to compare the segmentation performances of the two methods using the same conditions, we did not use the image datasets which were obtained by cropping the relevant regions-ofinterest [16]. Secondly, we did not perform post-image processing. Third, the number of test sets was only ten. Finally, the limitation associated with the use of our deep learning network, was based on the fact that the CT image was a three-dimensional (3D)-volume matrix, and each two-dimensional (2D) image was structurally connected to the previous image. However, DCNN does not take into account this structural connectivity because it uses a 2D convolution filter. All these factors may affect the performance of the auto-segmentation process. In post-image processing, Kim et al. [35] showed that the accuracy of the predicted contouring may vary differs according to the smoothing level of the contouring boundary surface. However, it would be 


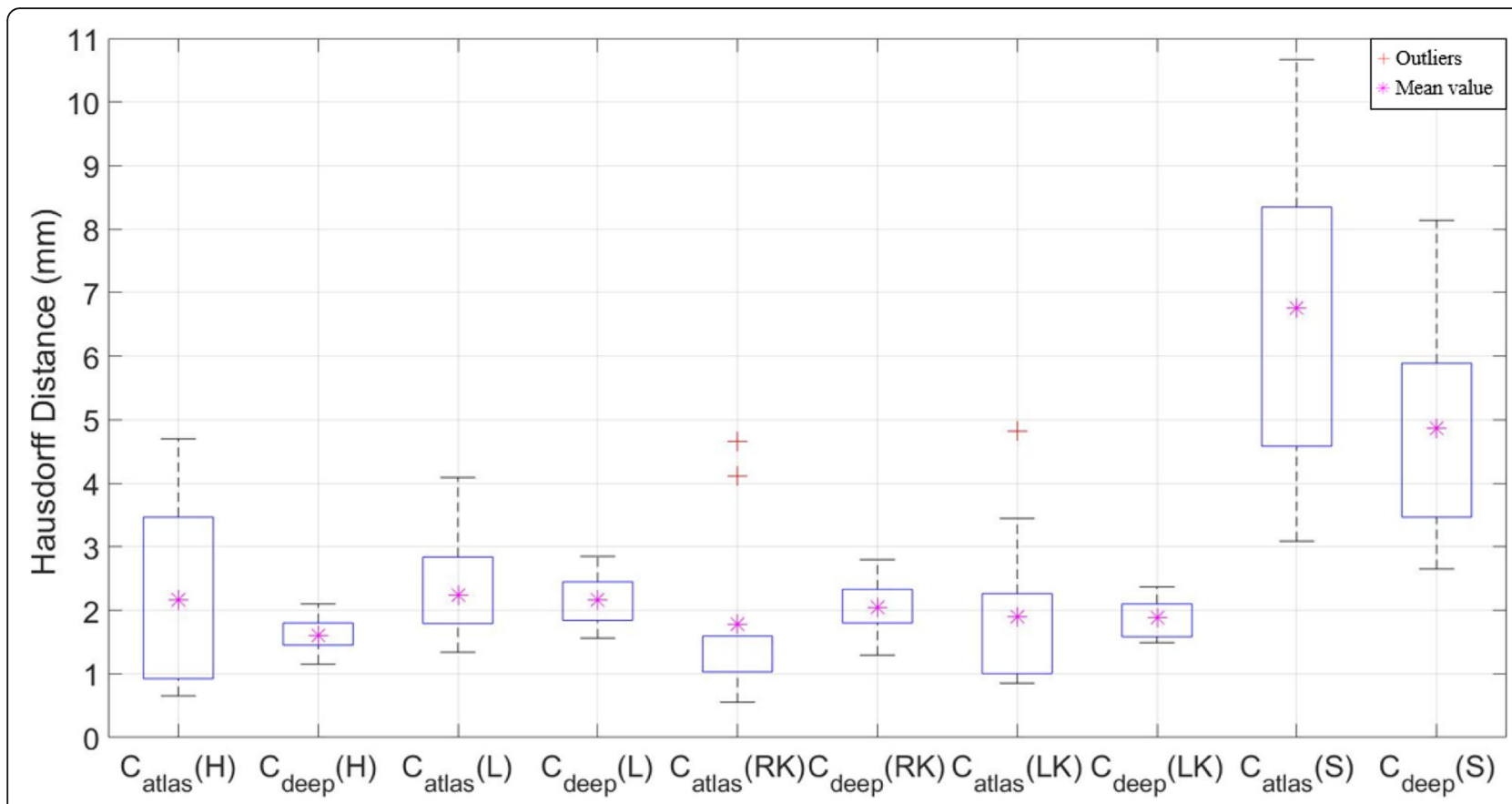

Fig. 6 Comparison of Dice similarity coefficient (DSC) value of deep learning contour $\left(C_{\text {deep }}\right)$ and atlas-based contour $\left(C_{\text {atlas }}\right)$ segmentations for the heart $(\mathrm{H})$, liver $(\mathrm{L})$, right kidney (RK), left kidney $(\mathrm{LK})$, and stomach $(\mathrm{S})$

difficult to represent statistically significant data for all clinical cases using such a small test dataset. In addition, recent studies have used 3D convolution filters to perform medical image segmentation. Milletari et al. [36] performed volumetric segmentation of magnetic resonance $(\mathrm{MR})$ prostate images with a $3 \mathrm{D}$ volumetric $\mathrm{CNN}$, an average dice score of $0.87 \pm 0.03$ and an average HD of $5.71 \pm 1.02 \mathrm{~mm}$.

The HD exhibited a difference in accuracy which depended on the image size. The size of the CT and

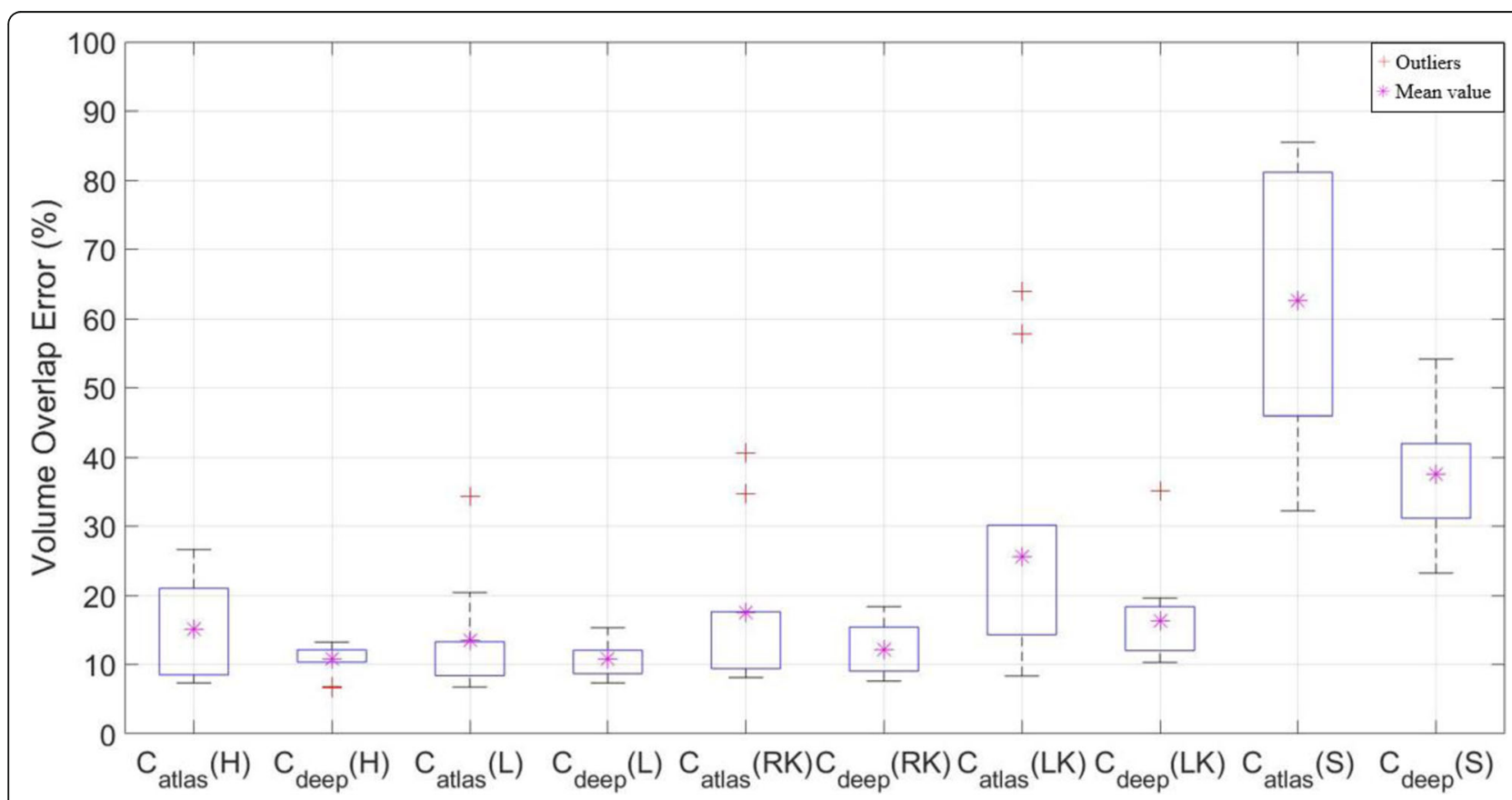

Fig. 7 Comparison of volume overlap error (VOE) for deep learning contour $\left(C_{\text {deep }}\right)$ and atlas-based contour $\left(C_{\text {atlas }}\right)$ segmentations for the heart $(\mathrm{H})$, liver (L), right kidney (RK), left kidney (LK), and stomach $(\mathrm{S})$ 


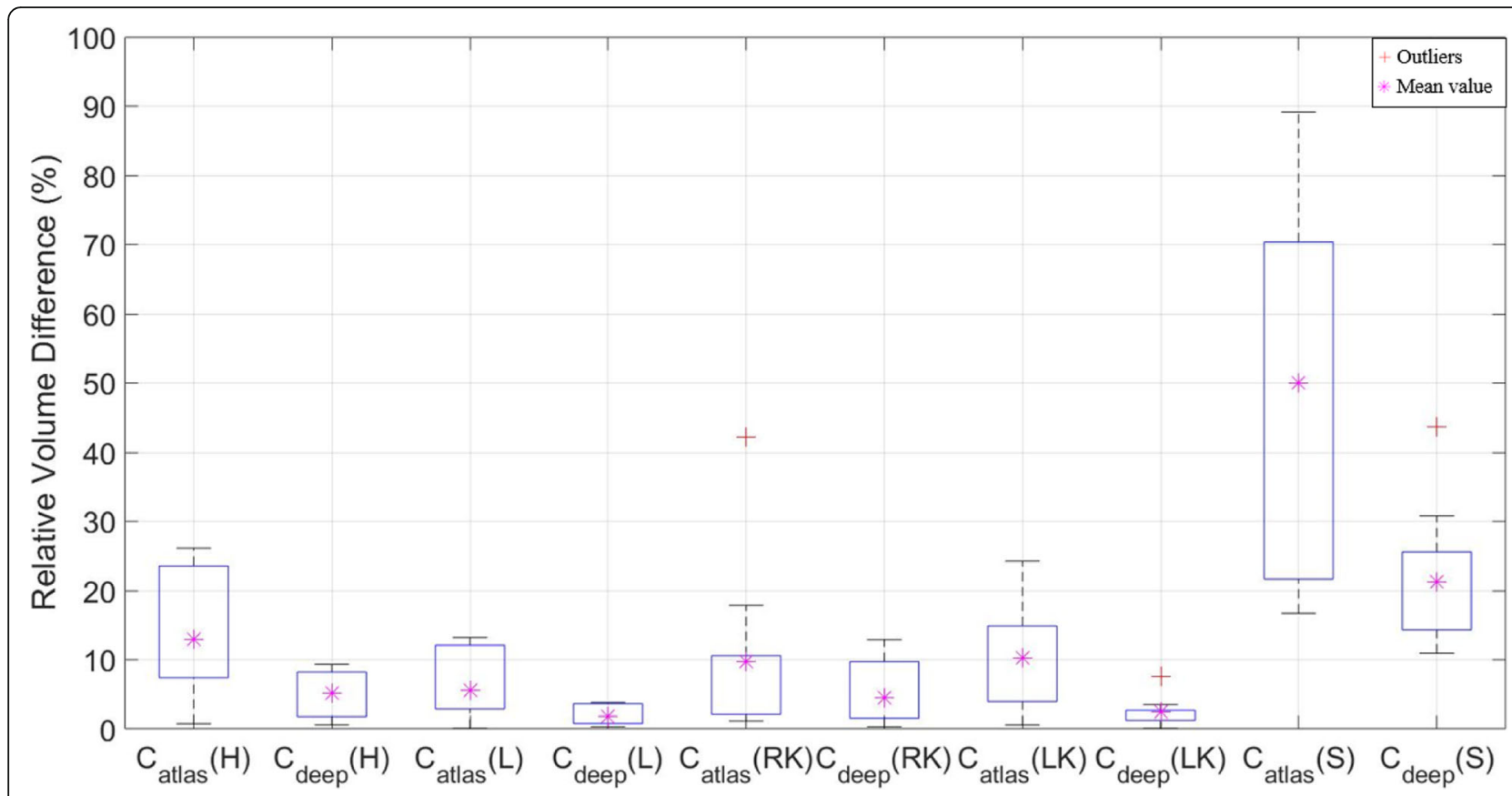

Fig. 8 Comparison of relative volume difference (RVD) for deep learning contour $\left(C_{\text {deep }}\right)$ and atlas-based contour $\left(C_{a t l a s}\right)$ segmentations for the heart $(H)$, liver (L), right kidney (RK), left kidney (LK), and stomach $(S)$

segmented labeled images were reduced to half the original sizes (i.e., to $256 \times 256$ ) because of the limitations of the graphic card memory and training time constraints. The standard deviations (SD) of the HD results after image interpolation to the matrix sizes of $64 \times 64$ pixels, $128 \times 128$ pixels, and $512 \times 512$ pixels, compared to the current pixels array size $256 \times 256$ pixels were \pm $0.63 \mathrm{~mm}, \pm 0.58 \mathrm{~mm}, \pm 0.97 \mathrm{~mm}, \pm 0.90 \mathrm{~mm}$, and \pm 1.03 $\mathrm{mm}$ for the heart, liver, right kidney, left kidney, and stomach, respectively. Accordingly, when the segmentation image size is changed, the HD result may yield a difference up to approximately $1 \mathrm{~mm}$.

However, comparison of the SD of the HD results of the current pixel array size $(256 \times 256)$ and the original CT pixel array size $(512 \times 512$ pixels $)$ yielded differences which were equal to $\pm 0.02 \mathrm{~mm}, \pm 0.04 \mathrm{~mm}, \pm 0.04 \mathrm{~mm}$, $\pm 0.07 \mathrm{~mm}$, and \pm 0.08 , in the cases of the heart, liver, right kidney, left kidney, and stomach, respectively.

Despite the aforementioned limitations, in this study, we compared the auto segmentation outcomes obtained with the use of the atlas, which is the auto segmentation tool currently used in clinical practice, with the use of

Table 8 Differences between DSC mean values associated with the deep-learning and atlas-based contouring methods

\begin{tabular}{lllllll}
\hline \multicolumn{2}{l}{ Subject organs } & Heart & Liver & Right Kidney & Left Kidney & Stomach \\
\hline DSC & $C_{\text {deep }}$ & 0.94 & 0.93 & 0.88 & 0.86 & 0.73 \\
& $C_{\text {atlas }}$ & 0.92 & 0.93 & 0.86 & 0.85 & 0.60 \\
1- $\left(C_{\text {deep }} / C_{\text {atlas }}\right)(\%)$ & -2.17 & 0 & -2.33 & -1.18 & -21.67 \\
\hline
\end{tabular}

an open source-based tool [21] rather than the commercial program [20].

In particular, HD is a sensitive index which indicates whether segmentation yields localized disagreements. Therefore, it is an important indicator for assessing the accuracy of the segmented boundaries. Considering the limitation of the SD differences based on pixel array size differences (comparison of the array sizes of $256 \times 256$ and $512 \times 512$ ) mentioned above, the deep-learningbased contouring is superior to the atlas-based contouring method regarding the HD results.

The segmentation results of the heart, liver, kidney, and stomach, based on the use of the auto-segmentation with deep-learning-based contouring showed good performance outcomes both in terms of DSC and HD compared to the atlas-based contouring. Loi et al. [37] proposed a sufficient DSC threshold $>0.85$ for volumes greater than $30 \mathrm{ml}$ for auto-segmentations. In this study, the vast majority met this criterion except in the case of the stomach, whereby only one of the test sets yielded DSC values greater than 0.85 in the case where, the deep learning method was used (Table 3).

Recent technological developments in diagnostic imaging modalities have led to frequent fusions of images, including the paradigms of MR-Linac, PET-CT, and MR-CT image fusions. To apply this to adaptive RT, efficient OAR delineation is necessary in the daily adaptive treatment protocol to minimize the total treatment time.

There is one important issue that needs to be considered to contour the OARs correctly, which pertains to 
Table 9 Differences between VOE mean values associated with the deep-learning-based and atlas-based contouring methods

\begin{tabular}{lllllll}
\hline \multicolumn{2}{l}{ Subject organs } & Heart & Liver & Right Kidney & Left Kidney & Stomach \\
\hline VOE (\%) & $C_{\text {deep }}$ & 10.84 & 10.82 & 12.19 & 16.31 & 37.53 \\
& $C_{\text {atlas }}$ & 15.17 & 13.51 & 17.51 & 25.63 & 62.64 \\
$C_{\text {deep }}-C_{\text {atlas }}(\%)$ & -4.33 & -2.69 & -5.32 & -9.32 & -25.11 \\
\hline
\end{tabular}

the motion artifacts attributed to the respiratory motion of the patients. The movement of the organ increases the contour uncertainty of the OARs. Combining the auto-segmentation with the reduction of motion artifacts [38] will enable more accurate delineation of the organs affected by respiration. Therefore, application of deeplearning-based auto-segmentation possesses tremendous potential, and is expected to have a greater impact in the near future in achieving effective and efficient radiotherapy workflow.

\section{Conclusions}

In summary, we applied an open-source, deep learning framework to an auto-segmentation application in liver cancer and demonstrated its performance improvements compared to the atlas-based approach. Deep-learningbased auto-segmentation is considered to yield an acceptable accuracy as well as good reproducibility for clinical use. Additionally, it can significantly reduce the contouring time in OARs destined to undergo radiation treatment planning. We envisage that deep learning-based autosegmentation will become clinically useful, especially when it is applied in the daily adaptive plans which are based on multi-imaging modality-guided treatments.

\section{Abbreviations}

CNN: Convolutional neural network; CT: Computer tomography; DCNN: Deep convolution neural network; DSC: Dice similarity coefficient; HD: Hausdorff distance; MV: Majority vote; OARs: Organs at risk; ReLu: Rectified linear unit; RT: Radiotherapy; RVD: Relative volume difference; SD: Standard deviation; VOE: Volume overlap error

\section{Acknowledgments}

Not applicable.

\section{Authors' contributions}

SHA, AUY, and JHJ conceived the study, participated in its design and coordination and helped draft the manuscript. THK, SHY, and ESO, generated the manual contours. SHA, KHK, CK, YG, SC, SBL, YKL, HK, DS, and TK, analyzed parts of the data, and interpreted the data. SHA developed and

Table 10 Differences between RVD mean values associated with the deep-learning-based and atlas-based contouring methods

\begin{tabular}{lllllll}
\hline Subject organs & Heart & Liver & Right Kidney & Left Kidney & Stomach \\
\hline RVD (\%) & $C_{\text {deep }}$ & 5.17 & 1.86 & 4.53 & 2.45 & 21.26 \\
& $C_{\text {atlas }}$ & 12.90 & 5.56 & 9.75 & 10.23 & 50.06 \\
$C_{\text {deep }}-C_{\text {atlas }}(\%)$ & -7.73 & -3.70 & -5.22 & -7.78 & -28.80 \\
\hline
\end{tabular}

designed the software and wrote the technical part. All authors read and approved the final manuscript.

\section{Funding}

This study was supported by the National Cancer Center Grant (1810273).

\section{Availability of data and materials}

The data are not available for public access because of patient privacy concerns, but are available from the corresponding author on reasonable request.

\section{Ethics approval and consent to participate}

This study was approved by our institutional review board and conducted in accordance with the ethical standards of the Declaration of Helsinki.

\section{Consent for publication}

Not applicable.

\section{Competing interests}

The authors declare that they have no competing interest.

\section{Author details}

${ }^{1}$ Department of Radiation Oncology, Proton Therapy Center, National Cancer Center, 323, Ilsan-ro, Ilsandong-gu, Goyang-si, Gyeonggi-do 10408, South Korea. ${ }^{2}$ Peter MacCallum Cancer Centre, Melbourne, VIC, Australia.

${ }^{3}$ Department of Radiation Oncology, Asan Medical Center, Seoul, South Korea. ${ }^{4}$ Department of Radiation Oncology, Chonnam National University Medical School, Gwangju, South Korea.

Received: 22 April 2019 Accepted: 9 October 2019

Published online: 27 November 2019

\section{References}

1. Vinod SK, Jameson MG, Min M, Holloway LC. Uncertainties in volume delineation in radiation oncology: a systematic review and recommendations for future studies. Radiother Oncol. 2016;121(2):169-79.

2. Chen A, Deeley MA, Niermann KJ, Moretti L, Dawant BM. Combining registration and active shape models for the automatic segmentation of the lymph node regions in head and neck CT images. Med Phys. 2010;37(12): 6338-46.

3. Qazi AA, Pekar V, Kim J, Xie J, Breen SL, Jaffray DA. Auto-segmentation of normal and target structures in head and neck CT images: a feature-driven model-based approach. Med Phys. 2011;38(11):6160-70.

4. Xu Y, Xu C, Kuang X, Wang H, Chang El, Huang W, et al. 3D-SIFT-flow for atlas-based CT liver image segmentation. Med Phys. 2016;43(5):2229-41.

5. Daisne J-F, Blumhofer A. Atlas-based automatic segmentation of head and neck organs at risk and nodal target volumes: a clinical validation. Radiat Oncol. 2013;8(1):154.

6. Sjöberg C, Lundmark M, Granberg C, Johansson S, Ahnesjö A, Montelius A. Clinical evaluation of multi-atlas based segmentation of lymph node regions in head and neck and prostate cancer patients. Radiat Oncol. 2013; 8(1):229.

7. Thomson D, Boylan C, Liptrot T, Aitkenhead A, Lee L, Yap B, et al. Evaluation of an automatic segmentation algorithm for definition of head and neck organs at risk. Radiat Oncol. 2014;9(1):173.

8. Karabatak M, Ince MC. An expert system for detection of breast cancer based on association rules and neural network. Expert Syst Appl. 2009; 36(2):3465-9.

9. Bejnordi BE, Veta M, Van Diest PJ, Van Ginneken B, Karssemeijer N, Litjens G, et al. Diagnostic assessment of deep learning algorithms for detection of lymph node metastases in women with breast cancer. JAMA. 2017:318(22):2199-210.

10. Übeyli ED. Implementing automated diagnostic systems for breast cancer detection. Expert Syst Appl. 2007;33(4):1054-62.

11. Sahiner B, Pezeshk A, Hadjiiski LM, Wang X, Drukker K, Cha KH, et al. Deep learning in medical imaging and radiation therapy. Med Phys. 2019:46(1):e1-e36.

12. Kang J, Schwartz R, Flickinger J, Beriwal S. Machine learning approaches for predicting radiation therapy outcomes: a clinician's perspective. Int J Radiat Oncol Biol Phys. 2015;93(5):1127-35. 
13. Poynton M, Choi B, Kim Y, Park I, Noh G, Hong S, et al. Machine learning methods applied to pharmacokinetic modelling of remifentanil in healthy volunteers: a multi-method comparison. J Int Med Res. 2009;37(6):1680-91.

14. LeCun Y, Bengio Y, Hinton G. Deep learning. Nature. 2015;521(7553):436.

15. Krizhevsky A, Sutskever I, Hinton GE. Imagenet classification with deep convolutional neural networks. In: Advances in neural information processing systems; 2012. p. 1097-105.

16. Hu P, Wu F, Peng J, Liang P, Kong D. Automatic 3D liver segmentation based on deep learning and globally optimized surface evolution. Phys Med Biol. 2016;61(24):8676.

17. Dong H, Yang G, Liu F, Mo Y, Guo Y. Automatic brain tumor detection and segmentation using U-Net based fully convolutional networks. In: Annual conference on medical image understanding and analysis. Cham: Springer; 2017. p. 506-17.

18. Zhou X, Takayama R, Wang S, Hara T, Fujita H. Deep learning of the sectional appearances of 3D CT images for anatomical structure segmentation based on an FCN voting method. Med Phys. 2017;44(10): 5221-33.

19. Tong N, Gou S, Yang S, Ruan D, Sheng K. Fully automatic multi-organ segmentation for head and neck cancer radiotherapy using shape representation model constrained fully convolutional neural networks. Med Phys. 2018;45(10):4558-67.

20. Yoon HJ, Jeong YJ, Kang H, Jeong JE, Kang DY. Medical image analysis using artificial intelligence. Progress Med Phys. 2019;30(2):49-58.

21. Lustberg T, van Soest J, Gooding M, Peressutti D, Aljabar P, van der Stoep J, et al. Clinical evaluation of atlas and deep learning based automatic contouring for lung cancer. Radiother Oncol. 2018;126(2):312-7.

22. Keras CF. The Python deep learning library. In: Astrophysics Source Code Library; 2018.

23. Quan TM, Hildebrand DG, Jeong W-K. Fusionnet: A deep fully residual convolutional neural network for image segmentation in connectomics. 2016. arXiv preprint arXiv:1612.05360. https://arxiv.org/abs/1612.05360.

24. Ronneberger O, Fischer P, Brox T. U-net: Convolutional networks for biomedical image segmentation. In: In: international conference on medical image computing and computer-assisted intervention. Cham: Springer; 2015. p. 234-41.

25. Nair $V$, Hinton GE. Rectified linear units improve restricted boltzmann machines. In: Proceedings of the 27th international conference on machine learning (ICML-10); 2010. p. 807-14.

26. loffe S, Szegedy C. Batch normalization: Accelerating deep network training by reducing internal covariate shift. CoRR.-2015.-Vol. abs/1502.03167. http:// arxiv.org/abs/1502.03167.

27. Hinton GE, Srivastava N, Krizhevsky A, Sutskever I, Salakhutdinov RR. Improving neural networks by preventing co-adaptation of feature detectors. arXiv preprint arXiv:12070580. 2012. https://arxiv.org/abs/12 07.0580.

28. Roth HR, Shen C, Oda H, Oda M, Hayashi Y, Misawa K, Mori K. Deep learning and its application to medical image segmentation. Medical Imaging Technology. 2018;36(2):63-71.

29. Kingma DP, Adam BJ. A method for stochastic optimization. In: Proceedings of the 3rd International Conference for Learning Representations (ICLR 2015); 2015

30. Taha AA, Hanbury A. Metrics for evaluating 3D medical image segmentation: analysis, selection, and tool. BMC Med Imaging. 2015;15:29.

31. Sharp G, Fritscher KD, Pekar V, Peroni M, Shusharina N, Veeraraghavan H, et al. Vision 20/20: perspectives on automated image segmentation for radiotherapy. Med Phys. 2014;41(5):050902.

32. Christ PF, Ettlinger F, Grün F, Elshaera MEA, Lipkova J, Schlecht S, Rempfler M. Automatic liver and tumor segmentation of CT and MRI volumes using cascaded fully convolutional neural networks. arXiv preprint arXiv:1702. 05970; 2017.

33. La Macchia M, Fellin F, Amichetti M, Cianchetti M, Gianolini S, Paola V, et al. Systematic evaluation of three different commercial software solutions for automatic segmentation for adaptive therapy in head-and-neck, prostate and pleural cancer. Radiat Oncol. 2012;7(1):160.

34. Gibson E, Giganti F, Hu Y, Bonmati E, Bandula S, Gurusamy K, et al. Automatic multi-organ segmentation on abdominal $\mathrm{CT}$ with dense $\mathrm{V}$ networks. IEEE Trans Med Imaging. 2018;37(8):1822-34.

35. Kim H, Monroe JI, Lo S, Yao M, Harari PM, Machtay M, et al. Quantitative evaluation of image segmentation incorporating medical consideration functions. Med Phys. 2015;42(6 Part 1):3013-23.
36. Milletari F, Navab N, Ahmadi SA. V-net: fully convolutional neural networks for volumetric medical image segmentation. In: 2016 fourth international conference on 3D vision (3DV); 2016. p. 565-71. IEEE.

37. Loi G, Fusella M, Lanzi E, Cagni E, Garibaldi C, lacoviello G, et al. Performance of commercially available deformable image registration platforms for contour propagation using patient-based computational phantoms: a multi-institutional study. Med Phys. 2018;45(2):748-5.

38. Jiang $\mathrm{W}$, Liu Z, Lee $\mathrm{KH}$, Chen $\mathrm{S}, \mathrm{Ng}$ YL, Dou Q, et al. Respiratory motion correction in abdominal MRI using a densely connected U-Net with GANguided training. arXiv preprint arXiv:1906.09745. 2019.

\section{Publisher's Note}

Springer Nature remains neutral with regard to jurisdictional claims in published maps and institutional affiliations.
Ready to submit your research? Choose BMC and benefit from:

- fast, convenient online submission

- thorough peer review by experienced researchers in your field

- rapid publication on acceptance

- support for research data, including large and complex data types

- gold Open Access which fosters wider collaboration and increased citations

- maximum visibility for your research: over $100 \mathrm{M}$ website views per year

At BMC, research is always in progress.

Learn more biomedcentral.com/submissions 